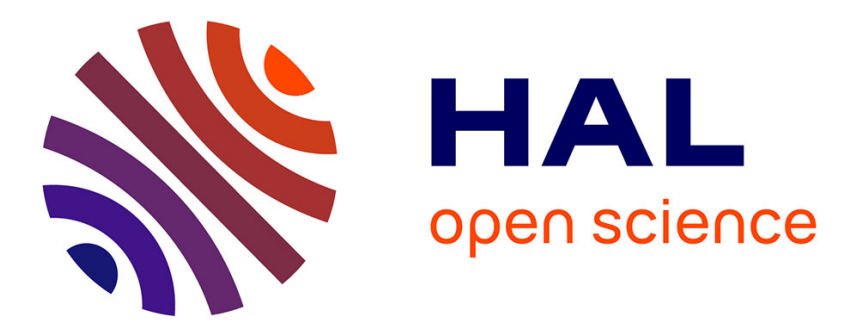

\title{
Design of Co/ Pd multilayer system with antiferromagnetic-to-ferromagnetic phase transition
}

Jan-Ulrich Thiele, Thomas Hauet, Olav Hellwig

\section{To cite this version:}

Jan-Ulrich Thiele, Thomas Hauet, Olav Hellwig. Design of $\mathrm{Co} / \mathrm{Pd}$ multilayer system with antiferromagnetic-to-ferromagnetic phase transition. Applied Physics Letters, 2008, 10.1063/1.2946654. hal-01345159

\section{HAL Id: hal-01345159 \\ https://hal.science/hal-01345159}

Submitted on 13 Jul 2016

HAL is a multi-disciplinary open access archive for the deposit and dissemination of scientific research documents, whether they are published or not. The documents may come from teaching and research institutions in France or abroad, or from public or private research centers.
L'archive ouverte pluridisciplinaire HAL, est destinée au dépôt et à la diffusion de documents scientifiques de niveau recherche, publiés ou non, émanant des établissements d'enseignement et de recherche français ou étrangers, des laboratoires publics ou privés. 


\title{
Design of Co/Pd multilayer system with antiferromagnetic-to-ferromagnetic phase transition
}

\author{
Jan-Ulrich Thiele, ${ }^{\text {a) }}$ Thomas Hauet, and Olav Hellwig \\ Hitachi Global Storage Technologies, San Jose Research Center, 3403 Yerba Buena Road, San Jose, \\ California 95135, USA
}

(Received 25 April 2008; accepted 28 May 2008; published online 17 June 2008)

\begin{abstract}
Among the known magnetic material systems, most are either purely antiferromagnetic or purely ferromagnetic at temperatures up to their critical temperature. There are only very few examples of materials that undergo a temperature dependent phase transition from an antiferromagnetic to a ferromagnetic phase or vice versa, and of these, only the chemically ordered alloy FeRh exhibits this transition near room temperature. Here we present a perpendicular anisotropy multilayer structure that by careful tuning of the constituent layers and their coupling mimics the magnetic behavior of FeRh, yet allows great flexibility in tuning its magnetic properties over a wide temperature range. (C) 2008 American Institute of Physics. [DOI: 10.1063/1.2946654]
\end{abstract}

The first order phase transition from an antiferromagnetic (AF) to a ferromagnetic (FM) phase in FeRh upon heating from room temperature to a transition temperature, $T_{\mathrm{AF}-\mathrm{FM}}$, of approximately $370 \mathrm{~K}$ was discovered in 1938 by Fallot. ${ }^{1} \quad$ In subsequent experiments using Mössbauer spectroscopy ${ }^{2}$ and neutron diffraction ${ }^{3}$ the spin structure of the two phases was shown to be collinear with moments of $3.2 \mu_{B}$ per Fe and $0.9 \mu_{B}$ per Rh atom for the FM phase and $3.3 \mu_{B}$ per Fe atom and no magnetic moment on the $\mathrm{Rh}$ for the AF phase. While other materials exhibiting similar first order AF-to-FM phase transitions such as $\mathrm{Ru}$-doped $\mathrm{CeFe}_{2}$ are known, ${ }^{4} \mathrm{FeRh}$ is unique in that the phase transition occurs around room temperature. The transition temperature can be tuned over a wide range by substitutional doping: it increases with small additions of Ir or Pt, and decreases with small additions of Pd or Ni. ${ }^{5}$ Recently, FeRh was investigated with renewed interest for potential applications in thermally assisted magnetic recording and memory devices. ${ }^{6}$ However, one of the drawbacks of FeRh is the high deposition or postannealing temperature required to obtain the chemically ordered phase.

The aim of the present study is to design a multilayer structure that mimics the behavior of FeRh, utilizing the great flexibility offered by magnetic multilayers to tune all relevant magnetic properties. ${ }^{7}$ The basic idea is to use two layers with large perpendicular magnetic anisotropy and high Curie temperatures $T_{C}$ separated by a layer providing AF coupling, and a layer with perpendicular anisotropy with a lower Curie temperature $T_{C \text {,interlayer }}$ in the range of the desired AF-FM transition temperature $T_{\mathrm{AF}-\mathrm{FM}}$. At room temperature, this system behaves as two antiferromagnetically coupled layers with a low net perpendicular magnetic moment in remanence. As the temperature is raised to approach $T_{C \text {,interlayer, }}$, the magnetization of the interlayer is gradually reduced to zero, and consequently the coupling strength provided by the combination of this low- $T_{C}$ layer and the adjacent AF coupling layer is reduced. Eventually, the effective coupling between the two high- $K_{U}$, high- $T_{C}$ layers becomes

\footnotetext{
${ }^{a)}$ Present address: Seagate Technology, Research \& Technology Development, 47010 Kato Road, Fremont, CA 94538. Electronic mail: janulrich.thiele@seagate.com.
}

dominated by their dipolar fields, resulting in a parallel alignment of their moments at remanence and a net magnetic moment equal to the sum of the moments of the two high- $T_{C}$ layers.

To prove the concept described above, we deposited sequences of $\mathrm{Co} / \mathrm{Pd}$ and $\mathrm{CoNi} / \mathrm{Pd}$ multilayers, as they allow great flexibility in adjusting the relevant parameters such as anisotropy, magnetization, Curie temperature, and intralayer as well as interlayer exchange coupling. The basic structure, as shown in Fig. 1, consists of two Co/Pd multilayers with large perpendicular magnetic anisotropy and high $T_{C}$ separated by a Co/Ru $(7 \AA) / C o$ structure providing AF coupling and a $\mathrm{CoNi} / \mathrm{Pd}$ multilayer with perpendicular anisotropy and $T_{C \text {,interlayer }}$ in the range of the desired AF-FM transition temperature $T_{\mathrm{AF}-\mathrm{FM}}$. The multilayer samples were deposited on glass substrates by magnetron sputtering from element targets in a commercial sputter system. An Ar gas pressure of 1.4 mTorr was used for sputtering all layers except the topmost $\mathrm{Co} / \mathrm{Pd}$ multilayer stack. This last multilayer was deposited at 5 mTorr in order to achieve a somewhat larger coercivity compared to the previous layers. The deposition rates of all layers were in the range of $0.5-1 \AA / \mathrm{s}$. Typical film thicknesses and compositions of the constituting multilayers were $[\mathrm{Co}(5 \AA) / \mathrm{Pd}(7.5 \AA)]$ for the first high- $T_{C}$ multilayer, Co $(5 \AA) / \mathrm{Ru}(7 \AA) / \mathrm{Co}(5 \AA)$ for the $\mathrm{AF}$ coupling layer, $\left[\mathrm{Co}_{50} \mathrm{Ni}_{50}(2.5 \AA) / \mathrm{Pd}(12 \AA)\right]$ for the separation layer and $[\mathrm{Co}(3.5 \AA) / \mathrm{Pd}(7.5 \AA)]$ for the second high- $T_{C}$ multilayer.

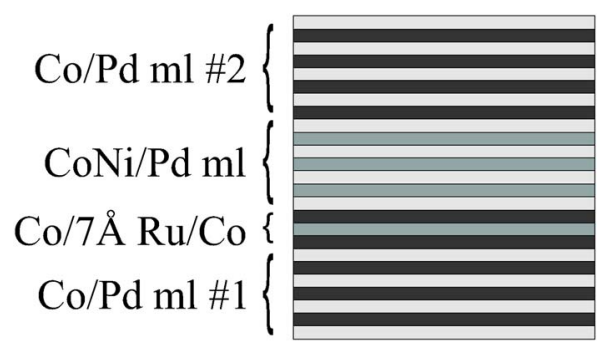

FIG. 1. (Color online) Sample structure showing the four basic building blocks: two Co/Pd multilayers with large perpendicular magnetic anisotropy and a high Curie temperature $T_{C}$, a Co/Ru $(7 \AA) / C o$ structure providing AF coupling, and a $\mathrm{CoNi} / \mathrm{Pd}$ multilayer with perpendicular anisotropy and $T_{C \text {,interlayer }}$ in the range of the desired AF-FM transition temperature $T_{\mathrm{AF}-\mathrm{FM}}$. 


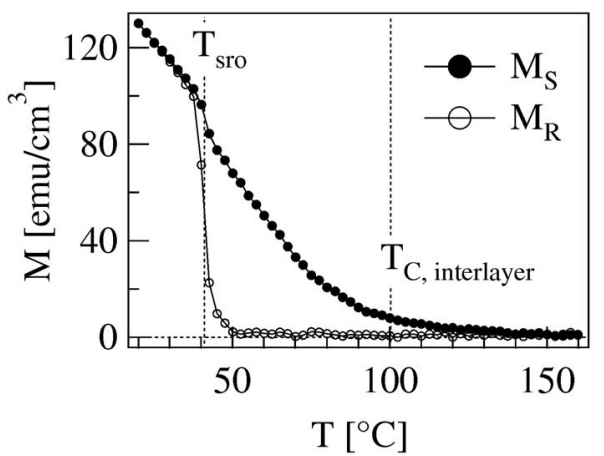

FIG. 2. Temperature dependence of the remanent magnetization $M_{R}$ and the saturation magnetization $M_{S}$ of a $\left[\mathrm{Co}_{50} \mathrm{Ni}_{50}(2.5 \AA) / \mathrm{Pd}(12 \AA)\right] \times 12$ multilayer. $T_{\text {sro }}$ denotes the temperature at which the effective anisotropy of the film changes from perpendicular to in-plane orientation $T_{C \text {,interlayer }}$ denotes the Curie temperature.

Thin seed and cap layers of about 50 and $25 \AA$ Pd were used. Film thicknesses were measured on calibration samples by grazing angle $\mathrm{x}$-ray diffraction, and the composition of the CoNi alloy used for the low- $T_{C}$ layer was verified using Rutherford backscattering. Hysteresis loops as well as temperature scans of all structures and the saturation magnetization $M_{S}$ and anisotropy constant $K_{1}$ of the constituent multilayers were measured in a maximum field of $2 \mathrm{~T}$ using a commercial vibrating sample magnetometer.

The two Co/Pd multilayers have an estimated $T_{C}$ of about $400-500{ }^{\circ} \mathrm{C}$ (it is actually difficult to measure $T_{C}$ experimentally, as at temperatures exceeding about $300{ }^{\circ} \mathrm{C}$ the individual layers of the multilayer start interdiffusing). ${ }^{8}$ In contrast, the $\mathrm{CoNi} / \mathrm{Pd}$ multilayer was tuned to obtain a $T_{C \text {,interlayer }}$ of approximately $100{ }^{\circ} \mathrm{C}$. Due to the more rapid decrease with temperature of the anisotropy relative to the magnetization, the $\mathrm{CoNi} / \mathrm{Pd}$ multilayer shows a spin reorientation from perpendicular to in-plane at a temperature $T_{\text {sro }}$ of about $40{ }^{\circ} \mathrm{C}$, as shown in Fig. 2. At room temperature, the complete multilayer system behaves as two antiferromagnetically coupled multilayers, resulting in two shifted hysteresis loops with a low net perpendicular magnetic moment in remanence. By proper choice of film thicknesses, compositions, and multilayer repeats the net moment at room temperature can be adjusted to zero, as shown in Fig. 3(a) for a $[\mathrm{Co} / \mathrm{Pd}] \times 5 / \mathrm{Co} / \mathrm{Ru} / \mathrm{Co} /[\mathrm{CoNi} / \mathrm{Pd}] \times 3 /[\mathrm{Co} / \mathrm{Pd}] \times 3$ sample. As the temperature of the sample is raised, at $T_{\text {sro }}$ first the effective perpendicular anisotropy of the $\mathrm{CoNi} / \mathrm{Pd}$ multilayer and subsequently at $T_{C \text {,interlayer }}$ its magnetization are reduced to zero. Without the mediation of the $\mathrm{CoNi} / \mathrm{Pd}$ multilayer, the coupling strength provided by the $\mathrm{Co} / \mathrm{Ru} / \mathrm{Co}$ layers is also greatly reduced and the effective coupling between the two $\mathrm{Co} / \mathrm{Pd}$ multilayers becomes dominated by their dipolar fields, resulting in a parallel orientation. Figure 3(b) shows the temperature dependence of the remanent magnetization, measured after applying a perpendicular field of $1 \mathrm{kG}$ at each temperature.

Interestingly, while $T_{C}$ of $\mathrm{CoNi} / \mathrm{Pd}$ multilayers generally does not vary with the number of repeats $N, T_{\mathrm{AF}-\mathrm{FM}}$ of the complete structure increases significantly with decreasing number of $\mathrm{CoNi} / \mathrm{Pd}$ repeats, as shown in Fig. 4 , for a series of $[\mathrm{Co} / \mathrm{Pd}] \times 5 / \mathrm{Co} / \mathrm{Ru} / \mathrm{Co} /[\mathrm{CoNi} / \mathrm{Pd}]$ $\times N /[\mathrm{Co} / \mathrm{Pd}] \times 3$ samples. This reflects the influence of the adjacent $\mathrm{Co}$ and $\mathrm{Co} / \mathrm{Pd}$ layers on the band structure and the resulting magnetic properties of the $\mathrm{CoNi} / \mathrm{Pd}$ multilayer as
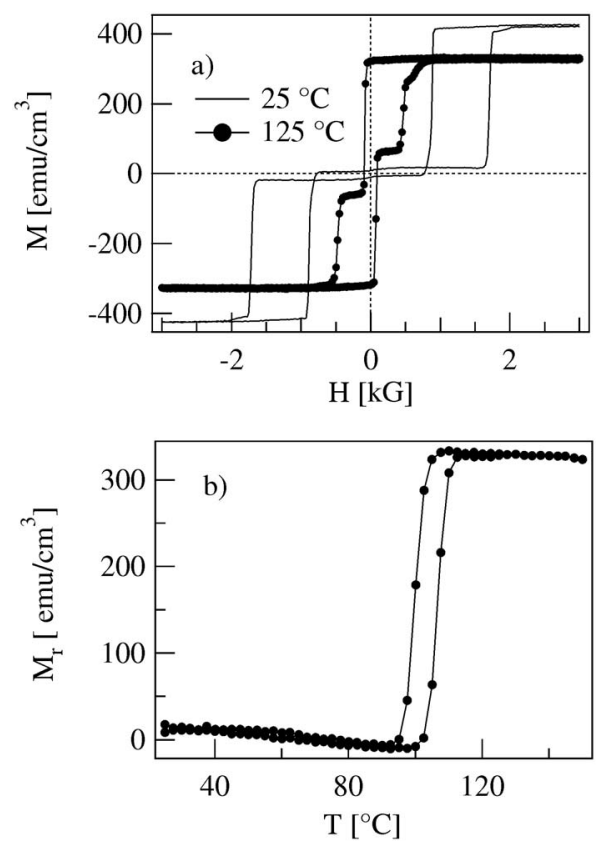

FIG. 3. (a) VSM hysteresis loops of a $[\mathrm{Co} / \mathrm{Pd}]$ $\times 5 / \mathrm{Co} / \mathrm{Ru} / \mathrm{Co} /[\mathrm{CoNi} / \mathrm{Pd}] \times 3 /[\mathrm{Co} / \mathrm{Pd}] \times 3$ sample measured at $25^{\circ} \mathrm{C}$ (line) and $125^{\circ} \mathrm{C}$ (symbols). (b) Remanent moment of that sample as a function of temperature, measured after applying a field of $1 \mathrm{kG}$ at each temperature.

described by Wang and Mills, ${ }^{9}$ and seen experimentally in studies of $\mathrm{FeF}_{2} / \mathrm{CoF}_{2}$ superlattices reported by Ramos et $a l .{ }^{10}$ Here a decay length of the same order as the exchange length of the material equivalent to approximately four repetitions, i.e., $l_{d}=6 \mathrm{~nm}$, can be estimated for this specific case from the data in Fig. 4. Moreover, for CoNi/Pd interlayers thicker than the decay length, $T_{\mathrm{AF}-\mathrm{FM}}$ converges to a value below $T_{C \text {,interlayer }}$ and slightly above $T_{\text {sro }}$, indicating that the spin reorientation is sufficient to trigger the AF-FM transition.

Finally, in the following, we discuss the similarities and differences between FeRh and the multilayer system presented here. Recently a unified theoretical description of $\mathrm{FeRh}$ and related alloys based on first-principles calculations for the exchange fields of the $\mathrm{Fe}$ atomic moment and the induced atomic moments on Rh was proposed. ${ }^{11}$ According to these calculations in both the AF and FM phases, the governing magnetic energy is the exchange energy, specifi-

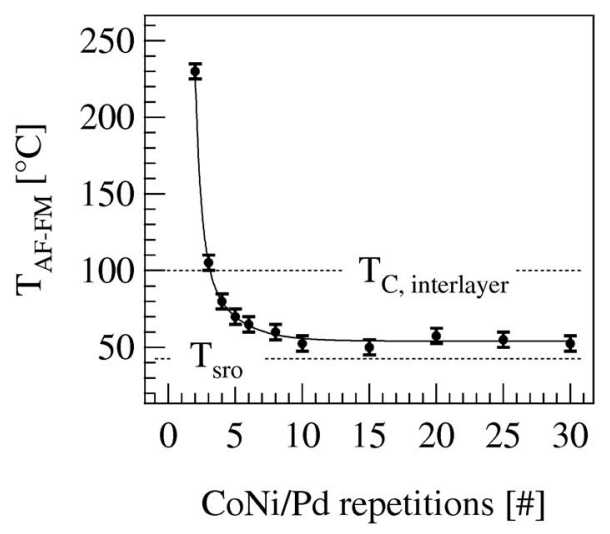

FIG. 4. AF-to-FM coupling transition temperature $T_{\mathrm{AF}-\mathrm{FM}}$, as a function of the number of repeats $N$ in the $\mathrm{CoNi} / \mathrm{Pd}$ multilayer in a series of $[\mathrm{Co} / \mathrm{Pd}]$ $\times 5 / \mathrm{Co} / \mathrm{Ru} / \mathrm{Co} /[\mathrm{CoNi} / \mathrm{Pd}] \times N /[\mathrm{Co} / \mathrm{Pd}] \times 3$. 
cally the moment induced by the $\mathrm{Fe}$ on the $\mathrm{Rh}$, and its interplay with the lattice spacing and the resulting band structure. In contrast, in the multilayer system presented here in the AF phase, it is the significantly weaker Ruderman-KittelKasuya-Yoshida (RKKY) interaction across the $\mathrm{Co} / \mathrm{Ru} / \mathrm{Co}$ coupling layer that results in the AF coupling and the zero net moment, while in the high temperature FM phase it is the dipolar fields of the two high $T_{C}$ multilayers that cause a parallel alignment. Consequently the energy scales governing the magnetic properties of the two materials are vastly different. At temperatures close to the AF-FM transition in FeRh, the transition can be induced by an external magnetic field, and the transition shifts linearly by about $8-9 \mathrm{~K} / \mathrm{T}$, ${ }^{12}$ i.e., for a sample with $T_{\mathrm{AF}-\mathrm{FM}}$ of approx $100^{\circ} \mathrm{C}$ a magnetic field of the order of $8 \mathrm{~T}$ is required to induce the phase transition at room temperature. In contrast, as shown in Fig. 3 , in the multilayer system presented here, only $0.18 \mathrm{~T}$ are required to overcome the RKKY coupling across the $\mathrm{Co} / \mathrm{Ru} / \mathrm{Co}$ coupling layer. Similarly, in the FM phase, FeRh is still governed by the exchange interaction, so the internal fields associated with, e.g., crystal defects are very large and accordingly in experimental samples no external fields are required to induce the AF-FM transition. In full films in a single-domain state dipolar fields are close to zero, so that in the multilayer system here small external fields are still required to induce the AF-FM transition even at temperatures above $T_{\mathrm{AF}-\mathrm{FM}}$. However, external fields are not required in samples in a multidomain state ${ }^{13}$ or nanostructured samples, such as, e.g., patterned media, one of the envisioned applications of such multilayer systems.

In summary, we have demonstrated a multilayer system that with increasing temperature shows a transition from antiparallel alignment with zero net remanent magnetic mo- ment to parallel alignment with a large net remanent magnetic moment. This is achieved by coupling two $\mathrm{Co} / \mathrm{Pd}$ multilayers via a $\mathrm{Co} / \mathrm{Ru}(7 \AA) / \mathrm{Co}$ structure providing $\mathrm{AF}$ coupling and a $\mathrm{CoNi} / \mathrm{Pd}$ multilayer with perpendicular anisotropy and tunable $T_{C}$ in the range of the desired AF-FM transition temperature. In terms of the temperature dependence of the remanent magnetization, this behavior mimics that of the natural supperlattice FeRh, which undergoes a first order phase transition from an $\mathrm{AF}$ to a FM phase.

T. Hauet was partially supported by a Lavoisier fellowship. We are grateful to Andrew Kellock for the Rutherford Backscattering measurements.

${ }^{1}$ M. Fallot, Ann. Phys. (Paris) 10, 291 (1938); M. Fallot and R. Horcart, Rev. Sci. 77, 498 (1939).

${ }^{2}$ G. Shirane, C. W. Chen, P. A. Flinn, and R. Nathans, J. Appl. Phys. 34, 1044 (1963).

${ }^{3}$ G. Shirane, C. W. Chen, and R. Nathans, Phys. Rev. 134, A1547 (1964). ${ }^{4}$ M. K. Chattopadhyay, S. B. Roy, and P. Chaddah, Phys. Rev. B 72, 180401 (2005); M. K. Chattopadhyay, S. B. Roy, A. K. Nigam, K. J. S. Sokhey, and P. Chaddah, ibid. 68, 174404 (2003).

${ }^{5}$ J. S. Kouvel, J. Appl. Phys. 37, 1257 (1966); J. S. Kouvel and C. C. Hartelius, ibid. 33, 1343 (1962).

${ }^{6}$ J.-U. Thiele, S. Maat, and E. E. Fullerton, Appl. Phys. Lett. 82, 2859 (2003).

${ }^{7}$ O. Hellwig, A. Berger, J. B. Kortright, and E. E. Fullerton, J. Magn. Magn. Mater. 319, 13 (2007).

${ }^{8}$ Y. H. Kim, A. K. Petford-Long, and J. P. Jakubovics, J. Appl. Phys. 76, 6099 (1994); P. Bruno, J. Magn. Soc. Jpn. 15, 15 (1991).

${ }^{9}$ R. W. Wang and D. L. Mills, Phys. Rev. B 46, 11681 (1992).

${ }^{10}$ C. A. Ramos, D. Lederman, A. R. King, and V. Jaccarino, Phys. Rev. Lett. 65, 2913 (1990).

${ }^{11}$ O. N. Mryasov, Phase Transitions 78, 197 (2005).

${ }^{12}$ S. Maat, J.-U. Thiele, and E. Fullerton, Phys. Rev. B 72, 214432 (2005).

${ }^{13}$ T. Hauet, C. Günther, B. Pfau, M. Schabes, J.-U. Thiele, R. L. Rick, P. Fischer, S. Eisebitt, and O. Hellwig, Phys. Rev. B 77, 184421 (2008). 